\title{
Estimator for the Heavy Tailed Index with the Stable Distribution
}

\author{
Hailong Chen, Yan Zhang and Fei Huang \\ School of Computer Science and Technology, \\ Harbin University of Science and Technology, \\ Harbin, China \\ hrbustchl@163.com
}

\begin{abstract}
Marginal distributions in the field of high frequency time series data of almost all type of heavy-tailed. It shows the importance of the study of heavy-tailed. Kesten's theorem combines Garch model parameters and stochastic equations to identify a property of a regular variation of GARCH model joint distributions. This paper has shown that finitedimensional joint distributions of GARCH model by Kesten are regularly varying distributions, and these distributions constitute heavy tailed distributions. We prove regular variation of the solution to the stochastic recurrent equation By Kesten's theorem, and the property of regular variation of finite joint distribution of the GARCH model has been proven, heavy tail index of ARCH(1) model distribution with stable residuals having $a \kappa$-stable distribution, $\varepsilon_{1}^{2} \sim S_{\kappa}(1,0,0)$. At last the nature of the relevant index estimates and conclusions are given.
\end{abstract}

Keywords: Garch Model; Heavy Tailed Distribution; Stochastic Equation

\section{Introduction}

The Heavy-tailed distribution characteristics prevalent in many areas, such as economy, finance, communications, hydrology and meteorology. For the study of heavy-tailed distributions first appeared in 1968, the British Gosset discovered tdistribution. Brodin [1] and Barabasi [2], Respectively, in the financial, weather, environment, human dynamics and other fields uses a heavy-tailed distribution. Due to the application of heavy-tailed distribution extends in all fields [3]. For heavy-tailed distribution studies, especially the tail index estimate became the focus of attention of scholars. Where Hill estimator [4], pickands estimator, moment estimation, and Kernel estimation is the most basic and most classical estimation methods. H. Kesten proposed a classic theory, in order to identify a property of a regular variation of GARCH model joint distributions. He only studied the regular variation of joint distributions. On this basis, this paper further research on the impact of the theorems for the heavy-tailed property.

The second part focuses on application to prove regular variation of the solution to the stochastic equation. These results are useful to prove properties of regular variation of GARCH model.

The third Part shows that finite-dimensional joint distributions of GARCH model are regularly varying distribution, and these distributions constitute tailed distributions. Prove the related theorems and conclusions, and gives the parameters of the estimation for index with heavy tailed distribution. 


\section{Regular Variation of the Solution to the Stochastic Equation}

This section will focus on application of H. Kesten's theorem [5] to prove regular variation of the solution to the stochastic equation. These results may be used to prove properties of regular variation of GARCH model.

Let us consider the scholastic equation:

$X_{t}=A_{t} X_{t-1}+B_{t}, t \in Z$

For a certain sequence $\left\{\left(A_{t}, B_{t}\right)\right\}$ of independent identically distributed $d \times d$ random matrices $A_{t}$ and $d$-dimensional random vectors $B_{t}, t \in Z$.

In general conditions, the stationary solution of the recurrent (1) satisfies the condition of multidimensional regular variation [5-6]. Let us consider the combination of theorems 3 and 4 in [5] as a modification of the fundamental result of H. Kesten:

\section{Theorem 1}

Let $\left\{\left(A_{t}, B_{t}\right)\right\}$ be independent identically distributed $d x d$ random matrices $A_{t}$ and $d$ dimensional random vectors with non-negative components $B_{t}, t \in Z$. Let us assume that the following conditions are met [5]:

a) when certain $\lambda>0, E\left\|A_{1}\right\|^{\lambda}<1$;

b) $A_{1}$ has almost sure non-zero lines;

c) a set $\left\{\ln \left\|a_{1} \ldots a_{n}\right\|: n \geq 1, a_{1} \ldots a_{n}>0\right.$ и $\left.\mathrm{a}_{1}, \ldots, a_{n} \in \operatorname{supp}\left(\mathrm{P}_{\mathrm{A}_{1}}\right)\right\}$ generates a dense group in $R$;

d) there exists the constant $k_{0}>0$, such that

$E\left(\min _{i=1, \ldots, d} \sum_{j=1}^{d} A_{i j}\right)^{k_{0}} \geq d^{k_{0} / 2}$

and

$E\left(\left\|A_{1}\right\|^{k_{0}} \ln ^{+}\left\|A_{1}\right\|\right)<\infty$.

Then we obtain:

1. There exists the unique solution $k_{1} \in\left(0, k_{0}\right]$ to the following equation.

$\lim _{n \rightarrow \infty} \frac{1}{n} \ln E\left\|A_{n} \ldots A_{1}\right\|^{k_{1}}=0$.

2. There exists the unique strictly stationary solution $\left\{X_{t}\right\}$ to the recurrent stochastic equation (1).

3. If $E\left|B_{1}\right|^{k_{1}}<\infty$, then the following condition of a regular variation is met for $X_{1}: \lim _{u \rightarrow \infty} u^{k_{1}} P\left(\left(x, X_{1}\right)>u\right)=w(x)$ which exists when all

$x \in R^{d} \backslash\{0\}$,

and $w(x)>0$ for all vectors $x$, all element of which are positive.

\section{Remark 1}

If $d=1$, then the condition of H. Kesten's theorem will be particularly simple. Indeed, if $A_{1}$ is a nonnegative random variable with a non-lattice distribution at $[0, \infty)$, 
$E \ln A_{1}<0, E A_{1}^{k_{0}} \geq 1$ and $E\left(A_{1}^{k_{0}} \ln ^{+} A_{1}\right)<\infty$, then all conditions of theorem 1 are met and (4) becomes the equation $E A_{1}^{k_{1}}=1$, which has the unique positive solution. If $E A_{1}^{k_{1}}<\infty$, then $X_{1}$ constitutes a regularly varying random variable with index $k_{1}$.

It is clear that (5) is a particular case, where a slowly varying function $L(x)$ is the positive constant. The below result is the direct consequence of theorem 1 .

\section{Consequence 1}

Let us assume that all conditions of theorem 1 are met, then the unique strictly stationary solution $\left\{X_{t}\right\}$ to the recurrent stochastic (1) is a regularly varying equation to mean the following: if $k_{1}$ in (4) constitutes an odd number, then there are the constant $C$ and a random vector $\Theta$ on the unit sphere $S^{d-1}$ such that

$$
u^{k_{1}} P(|X|>t u, X /|X| \in A) \stackrel{v}{\longrightarrow} C t^{-k_{1}} P(\Theta \in A), u \rightarrow \infty,
$$

for all Borel sets $A, A \subset S^{m-1}$ and $t>0$.

\section{Consequence 2}

Let us assume that conditions of consequence 1 occur, then finite-dimensional distributions of the stationary solution $\left\{X_{t}\right\}$ to the stochastic recurrent equation (1) are regularly varying distributions with index $k_{1}$.

We can rewrite this as

$$
\left(X_{1}, \ldots, X_{m}\right)=\left(A_{1}, A_{2} A_{1}, \ldots, A_{m} \ldots A_{1}\right) X_{0}+R_{m}
$$

where components of a random vector $R_{m}$ have lighter tails than components of a random vector $X_{0}$. Properties of a regular variation of a random vector may be found in study [7].

\section{Regular Variation of Joint Distribution of GARCH Model and Estimation of ARCH(1) Model Heavy Tail Index with Stable Residuals}

It is well known that regular variation of joint distributions ensures many properties of process moments and also the structure of correlation between observations. By inserting squares of $y_{t}^{2}$ and $\sigma_{t}^{2}$ of stationary GARCH $\left\{y_{t}\right\}$ model and its scedasticity $\left\{\sigma_{t}\right\}$ into stochastic equation, the classic theory of heavy tails may be used to solve the stochastic equation. This theory was developed by H. Kesten (1973), P. Embrechts, C. Kluppelberg, T. Mikosch (1997), C. M. Goldie (1991) in order to identify a property of a regular variation of GARCH model joint distributions. In this section we will show that finite-dimensional joint distributions of GARCH model are regularly varying distributions, i.e., these distributions constitute heavy tailed distributions.

Let us consider GARCH(p, q) model:

$$
y_{t}=\sigma_{t} \varepsilon_{t}, \sigma_{t}^{2}=\omega_{0}+\sum_{i=1}^{p} \alpha_{i} y_{t-i}^{2}+\sum_{j=1}^{q} \beta_{j} \sigma_{t-j}^{2}, t \in Z,
$$

where $\left\{\varepsilon_{t}, t \in Z\right\}$ are independent identically distributed random variables, $\omega_{0}>0$, $\alpha_{i} \geq 0,1 \leq i \leq p, \quad \beta_{j} \geq 0,1 \leq j \leq q$.

Let us rewrite the stochastic equation of GARCH model as follows:

$X_{t}=A_{t} X_{t-1}+B_{t}, t \in Z$, 
Where

$$
A_{n}=\left[\begin{array}{cccc}
\tau_{n} & \beta_{q} & \alpha & \alpha_{p} \\
I_{q-1} & 0 & 0 & 0 \\
\xi_{n} & 0 & 0 & 0 \\
0 & 0 & I_{p-2} & 0
\end{array}\right],
$$

and $\tau_{n}=\left(\beta_{1}+\alpha_{1} \varepsilon_{n}^{2}, \beta_{2}, \ldots, \beta_{q-1}\right) \in R^{q-1}, \xi_{n}=\left(\varepsilon_{n}^{2}, 0, \ldots, 0\right) \in R^{q-1}, \quad I_{n}$ is the unit matrix, $n=0,1,2, \ldots, \quad \alpha=\left(\alpha_{2}, \ldots, \alpha_{p-1}\right) \in R^{p-2}, \quad B_{t}=\left(\omega_{0}, 0, \ldots, 0\right)^{T} \in R^{p+q-1}, \quad$ and $X_{n}=\left(\sigma_{n}^{2}, . ., \sigma_{n-q-1}^{2}, y_{n-1}^{2}, \ldots, y_{n-p-1}^{2}\right)^{T} \in R^{p+q-1}$. It follows from the independence of sequence of residuals $\left\{\varepsilon_{t}\right\}$ that sequence $\left\{\left(A_{t}, B_{t}\right)\right\}$ is a sequence of independent $d \mathrm{x} d$ random matrices $A_{t}$ and $d$-dimensional random vectors $B_{t}$, where $d=p+q$.

Using theorem 1, we can make the following conclusion with respect to a regular variation of GARCH model joint distributions.

\section{Theorem 2}

Let us assume that the following conditions are met:

a) $\varepsilon_{1}$ has positive density at $R$, such that $E\left|\varepsilon_{1}\right|^{h}<\infty$ where all $h<h_{0}$ and $E\left|\varepsilon_{1}\right|^{h_{0}}=\infty$ at certain $h_{0} \in(0, \infty]$;

b) not all parameters of $\alpha_{i}$ and $\beta_{i}$ are zero. Then there is the constant $k_{1}>0$ and limited function $w(x)$, such that $\lim _{u \rightarrow \infty} u^{k_{1}} P\left(\left(x, X_{1}\right)>u\right)=w(x)$ exists where all $x \in R^{d} \backslash\{0\}$, i.e., $\left(x, X_{1}\right)$ constitutes a regularly varying random variable with index $k_{1}$. Besides, if $x \in[0, \infty)^{d} \backslash\{0\}$, then $w(x)>0$ and if $k_{1}$ is not even, then $X_{t}$ constitutes a regularly varying random variable with index $k_{1}$, i.e., there is a random vector $\Theta$ on a unit sphere $S^{p+q-2}$, such that

$u^{k_{1}} P(|X|>t u, X /|X| \in A) \stackrel{v}{\longrightarrow} t^{-k_{1}} P(\Theta \in A), u \rightarrow \infty$

For all Borel sets $A, A \subset S^{m-1}$.

Proof:

It follows from the first condition of the theorem that condition is met. Hence follows that there exists the unique strictly stationary solution of GARCH model. Let us consider subsequence $\tilde{X}_{t}=X_{t m}$ of sequence $\left\{X_{t}\right\}$ which is determined by the stochastic (7), for certain natural number $m$. After that, we will rewrite this as

$$
X_{t m}=A_{t m} \ldots A_{t(m-1)+1} X_{t(m-1)}+B_{t}+\sum_{k=1}^{\infty} A_{t m} \ldots A_{t m-k+1} B_{t m-k}=\tilde{A}_{t} X_{t(m-1)}+\tilde{B}_{t}, t \in Z,
$$

where $\left\{\left(\tilde{A}_{t}, \tilde{B}_{t}\right)\right\}$ is the independent identically distributed consequence. That is why for $\left\{\tilde{X}_{t}\right\}$ the following stochastic equation is valid

$$
\tilde{X}_{t}=\tilde{A}_{t} \tilde{X}_{t-1}+\tilde{B}_{t}, t \in Z \text {. }
$$

From the first condition of the theorem, the definition of Lyapunov's exponent, we obtain that if the value of $m$, is rather large there exists a rather small number $\lambda>0$, such that $E\left\|\tilde{A}_{1}\right\|^{\lambda}<1$ and $E\left|\tilde{B}_{1}\right|^{\lambda}<\infty$. It follows there from that the first condition of theorem 1 is met. 
Let us remark that components of a random matrix $\tilde{A}_{1}$ have multilinear expressions of random variables $\varepsilon_{t}^{2}$. Besides, from the condition $E\left|\varepsilon_{1}\right|^{h}<\infty$ where all $h<h_{0}$ and $E\left|\varepsilon_{1}\right|^{h_{0}}=\infty$ we obtain that $E\left|\varepsilon_{1}\right|^{h}$ take any large value when the value of $h$ is close to the value of $h_{0}$. From here we obtain that (2) is satisfied if $k_{0}$ has a rather large value and (3) is also satisfied where $h<h_{0}$.

Further we will show a property of natural numbers $\ln \left\|\tilde{a}_{1} \ldots \tilde{a}_{n}\right\|$, where values of $\tilde{a}_{i}$ lie in $\operatorname{supp}\left(\tilde{A}_{1}\right)$, generate dense group at $\mathrm{R}$. Indeed, from the fact that components of random matrix $\tilde{A}_{1}$ have multilinear expressions of random variables $\varepsilon_{t}^{2}$, which have density in $(0, \infty)$, we obtain that $\tilde{A}_{1}$ has all positive components. On the other hand, multilinear expressions of components of random matrix $\tilde{A}_{1}$ constitute continuous functions in $\varepsilon_{t}^{2}$, that is why the support $\tilde{A}_{1}$ is a connected set and the support $\left\|\tilde{A}_{1}\right\|$ is also a connected set. From here we obtain that if the value of $m$ is rather large the support $\ln \left\|\tilde{A}_{1}\right\|$ contains an interval which results in the density of natural numbers $\ln \left\|\tilde{a}_{1} \ldots \tilde{a}_{n}\right\|$.

Thus, for the stochastic (9) all conditions of theorem 1 are satisfied. From here we obtain a regular variation of a random variable $\left(x, X_{1}\right)$ with index $k_{1}>0$. If $k_{1}$ is an odd number, we will obtain (8) from consequence 1.

\section{Consequence 3}

Let conditions of theorem 2 be satisfied, then a distribution tail of a stationary GARCH model (6) varies as a power function: there exist the constant $k_{1}>0$ and positive constants $c_{|X|}$ and $c_{\sigma}$, such that

$$
P\left(\left|X_{1}\right|>x\right) \sim c_{|X|} x^{-2 k_{1}} \text { and } P\left(\left|\sigma_{1}\right|>x\right) \sim c_{\sigma} x^{-2 k_{1}} .
$$

Using the result of L. Breiman [5], we will obtain that

$$
P\left(\left|X_{1}\right|>x\right)=\frac{1}{2} P\left(\sigma_{1}\left|\varepsilon_{1}\right|>x\right) \sim E\left|\varepsilon_{1}\right|^{2 k_{1}} P\left(\sigma_{1}>x\right) \text {. }
$$

Let us assume that $\varepsilon_{1}$ is a symmetric random variable (that is why $X_{1}$ is also a symmetric random variable). Example: $\varepsilon_{1}$ has normal distribution or Student's tdistribution. Then $\operatorname{sign}\left(\varepsilon_{1}\right)$ and $\left|\varepsilon_{1}\right|$ are independent. From here we obtain that.

$$
P\left(X_{1}>x\right)=\frac{1}{2} P\left(\left|X_{1}\right|>x\right) \sim \frac{1}{2} E\left|\varepsilon_{1}\right|^{2 k_{1}} c_{\sigma} x^{-2 k_{1}} \text {. }
$$

In general, it is difficult to calculate values of $c_{|X|}, c_{\sigma}$ and index $k_{1}$, but in case of $\operatorname{ARCH}(1)$ and $\operatorname{GARCH}(1,1)$ these values may be calculated. For example, for $\operatorname{GARCH}(1,1)$ model, i.e., $\operatorname{GARCH}(1,1)$ model where $\alpha_{0}+\beta_{0}=1,\left\{\varepsilon_{t}\right\}$ have normal distribution, $E \varepsilon_{0}^{2}=1$. Then, equation (11) has the unique root $k=2$. Thus

$E \ln A_{0}<\ln E\left(\alpha_{0} \varepsilon_{0}^{2}+\beta_{0}\right)=0$, and $P\left(A_{0}>1\right)=P\left(\alpha_{0} \varepsilon_{0}^{2}+\beta_{0}>1\right)>0$.

From here, we obtain

$$
\begin{aligned}
& P\left(\sigma_{0}>x\right) \sim C_{0} x^{-2} \text { and } x \rightarrow \infty, \\
& P\left(\left|y_{0}\right|>x\right) \sim C_{0} x^{-2} \text { and } x \rightarrow \infty .
\end{aligned}
$$

Let us estimate heavy tailed distribution index $k_{1}$ of $\mathrm{ARCH}(1)$ model with stable residuals. 
Let us consider $\operatorname{GARCH}(1,1)$ process

$y_{t}=\sigma_{t} \varepsilon_{t}, \sigma_{t}^{2}=\omega_{0}+\alpha_{0} y_{t-1}^{2}+\beta_{0} \sigma_{t-1}^{2}, t \in Z$,

where $\left\{\varepsilon_{t}^{2}, t \in Z\right\}$ are independent identically distributed $\kappa$-stable random variables, $\kappa \in(0,2], \omega_{0}>0, \alpha_{0}>0, \beta_{0} \geq 0$.

Let $A_{0}=\beta_{0}+\alpha_{0} \varepsilon_{0}^{2}$. Let us examine properties of the behavior of distributions of $\operatorname{GARCH}(1,1)$ model and residuals $\left\{\varepsilon_{t}\right\}$. Relation between powers of $\operatorname{GARCH}(1,1)$ model tail, its coefficients and distribution of residuals is studied in paper [9]. In more detail please refer to [10-11]. As the conclusion from theorem 2 we obtain that if $E \ln A_{0}<0, P\left(A_{0}>1\right)>0$ and there is a constant $h_{0}>0$, such that $E A_{0}^{h}<\infty$ for all $h<h_{0}, E A_{0}^{h_{0}}=\infty$, the equation

$$
E A_{0}^{k / 2}=1
$$

Has the unique positive root $k=k_{1}$, then model (10) has the unique stationary solution. Besides, there exists the positive constant $C_{0}$, such that

$$
P\left(\sigma_{0}>x\right) \sim C_{0} x^{-k_{1}} \text { при } x \rightarrow \infty
$$

and

$$
P\left(\left|y_{0}\right|>x\right) \sim E\left|\varepsilon_{0}\right|^{k_{1}} P\left(\sigma_{0}>x\right) \text { при } x \rightarrow \infty
$$

When $\left\{\varepsilon_{t}\right\}$ are $\kappa$-stable random variables, $\kappa \in(0 ; 2]$, then the first condition of theorem 2 is met. That is why in this case conditions of D. Nelson necessary and sufficient for existence of the unique stationary solution to $\operatorname{GARCH}(1,1)$ model $(10)$ are always met. Besides, it follows from the property of the moment of stable distributions that equation (11), where $\varepsilon_{0}^{2}$ is a $\kappa$-stable random variable, has unique positive root which is smaller than $\kappa$. From here we obtain that if squares of residuals constitute $\kappa$-stable random variables, then the nature of behavior of heavy tails (12) and (13) of GARCH(1,1) model is satisfied, and behavior of model tails is heavier than the behavior of tails of its residuals.

In this section we will write $X \sim S_{\kappa}(\sigma, \beta, \mu), \kappa \in(0,2], \sigma>0, \beta \in[-1,1], \mu \in R$, if $X$ is a $\kappa$-stable random variable with a characteristic function in the form of stable distribution.

\section{Theorem 3}

If $X \sim S_{\kappa}(\sigma, \beta, 0), \kappa \in(0,2]$, then if all $p, 0<p<\kappa$, we obtain that

$$
E|X|^{p}=\frac{2 \Gamma\left(1-\frac{p}{\kappa}\right)}{\Gamma(1-p)} \cos \left(\gamma p \frac{\pi}{\kappa}\right), \text { where } \gamma=\beta K(\kappa) .
$$

Proof:

Let $q_{X}(x, \kappa, \beta)$ denote a function of the $X$ distribution density. Thus, if $\kappa \neq 1$, then

$q_{X}(-x, \kappa,-\beta)=\int_{R} e^{i x t} \phi_{X}(t) d t=\int_{R} e^{i x t} \exp \left\{-|t|^{\kappa} \exp \left[i \beta \operatorname{sign}(t) \frac{\pi}{2} K(\kappa)\right]\right\} d t=$ 


$$
\int_{+\infty}^{-\infty} e^{-i x t} \exp \left\{-|t|^{\kappa} \exp \left[-i \beta \operatorname{sign}(t) \frac{\pi}{2} K(\kappa)\right]\right\} d t=q_{X}(x, \kappa, \beta)
$$

In a similar way we will obtain the result (14) for the case where $\kappa=1$. That is why in order to obtain order $p$ raw moment it is sufficient to find representations for $q_{X}(x, \kappa, \beta)$, when $x>0$.

We obtain that

$$
\begin{aligned}
q_{X}(-x, \kappa,- & \beta)=q_{X}(x, \kappa, \beta)=\frac{1}{\pi} \operatorname{Re} \int_{R} e^{i x t} \phi_{X}(t) d t \\
= & \frac{1}{\pi} \operatorname{Re} \int_{0}^{\infty} e^{-i x t} \exp \left\{-t^{\kappa} e^{-i \beta \pi K(\kappa) / 2}\right\} d t=\frac{1}{\pi} \operatorname{Re} \int_{0}^{\infty} \exp \left\{-i t x-t^{\kappa} e^{-i \gamma \pi / 2}\right\} d t
\end{aligned}
$$

Where $\gamma=\beta K(\kappa)$.

Using Jordan's lemma, we will obtain

$$
\int_{0}^{\infty} \exp \left\{-i t x-t^{\kappa} e^{-i \gamma \pi / 2}\right\} d t=-i \int_{0}^{\infty} \exp \left\{-t x-t^{\kappa} e^{-i \rho \pi}\right\} d t \text {, where } \rho=\frac{\gamma+\kappa}{2} \text {. }
$$

We will obtain there from

$$
q_{X}(x, \kappa, \beta)=\frac{1}{\pi} i m \int_{0}^{\infty} \exp \left\{-t x-t^{\kappa} e^{-i \rho \pi}\right\} d t .
$$

Then

$$
\begin{aligned}
I_{X}(p, \kappa, \beta) & =\int_{0}^{\infty} x^{p} q_{X}(x, \kappa, \beta) d t=\frac{1}{\pi} \int_{0}^{\infty} x^{p} \operatorname{im} \int_{0}^{\infty} \exp \left\{-t x-t^{\kappa} e^{-i \rho \pi}\right\} d t d x \\
& =\frac{1}{\pi} \int_{0}^{\infty} x^{p} e^{-t x} d x \operatorname{im} \int_{0}^{\infty} \exp \left\{-t^{\kappa} e^{-i \rho \pi}\right\} d t=\frac{1}{\pi} \int_{0}^{\infty}(t x)^{p} e^{-t x} d(t x) t^{-(p+1)} \operatorname{im} \int_{0}^{\infty} \exp \left\{-t^{\kappa} e^{-i \rho \pi}\right\} d t \\
& =\frac{1}{\pi} \Gamma(p+1) \operatorname{im} \int_{0}^{\infty} t^{-(p+1)} \exp \left\{-t^{\kappa} e^{-i \rho \pi}\right\} d t .
\end{aligned}
$$

Let $z=t^{\kappa} e^{-i \rho \pi}$, then $t=z^{1 / \kappa} e^{-i \rho \pi / \kappa}, d z=\kappa t^{\kappa-1} e^{-i \rho \pi} d t$.

From here it follows that if all $p<\kappa$

$$
\begin{aligned}
& I_{X}(p, \kappa, \beta)=\frac{1}{\pi} \Gamma(p+1) \operatorname{im} \int_{0}^{\infty} \frac{1}{\kappa} t^{-(p+1)} e^{-z} t^{1-\kappa} e^{-i \rho \pi} d t \\
&=\frac{1}{\kappa \pi} \Gamma(p+1) \operatorname{im} \int_{0}^{\infty} z^{-(p+\kappa) / \kappa} e^{-i \rho \pi p / \kappa} e^{-z} d t \\
&=\frac{1}{\kappa \pi} \Gamma(p+1) \operatorname{im}\left(e^{-i \rho \pi p / \kappa}\right) \int_{0}^{\infty} z^{-p / \kappa-1} e^{-z} d t \\
&=-\frac{1}{\kappa \pi} \Gamma(p+1) \sin \left(\frac{\rho \pi p}{\kappa}\right) \Gamma\left(-\frac{p}{\kappa}\right)= \\
& \frac{1}{p \pi} \sin \left(\frac{\rho \pi p}{\kappa}\right) \Gamma(p+1) \Gamma\left(1-\frac{p}{\kappa}\right) .
\end{aligned}
$$


From(14) and (15) we obtain

$$
\begin{aligned}
& E|X|^{p}=\int_{-\infty}^{+\infty}|x|^{p} q(p, \kappa, \beta) d x=\int_{-\infty}^{0}(-x)^{p} q_{X}(x, \kappa, \beta) d x+\int_{0}^{+\infty}(x)^{p} q_{X}(x, \kappa, \beta) d x= \\
= & \int_{0}^{+\infty}(x)^{p} q_{X}(-x, \kappa, \beta) d x+\int_{0}^{+\infty}(x)^{p} q_{X}(p, \kappa, \beta) d x=\int_{0}^{+\infty}(x)^{p} q_{X}(x, \kappa,-\beta) d x+\int_{0}^{+\infty}(x)^{p} q_{X}(p, \kappa, \beta) d x \\
= & I_{X}(p, \kappa,-\beta)+I_{X}(p, \kappa, \beta)=\frac{1}{p \pi} \Gamma(p+1) \Gamma\left(1-\frac{p}{\kappa}\right)\left[\sin \left(\frac{(\kappa-\gamma) p}{\kappa} \pi\right)+\sin \left(\frac{(\kappa+\gamma) p}{\kappa} \pi\right)\right] \\
= & \frac{2 \Gamma\left(1-\frac{p}{\kappa}\right)}{\Gamma(1-p)} \cos \left(\gamma p \frac{\pi}{\kappa}\right) .
\end{aligned}
$$

The theorem has been proven.

Using theorem 3 and (11) we obtain that the index of the regular variation of ARCH(1) model with residuals having a $\kappa$-stable distribution, $\varepsilon_{1}^{2} \sim S_{\kappa}(1,0,0)$

$$
y_{t}=\sigma_{t} \varepsilon_{t}, \sigma_{t}^{2}=\omega_{0}+\alpha_{0} y_{t-1}^{2}, t \in Z
$$

is the root $k=k_{1}$ of the following equation

$$
\left(\alpha_{0}\right)^{k} \frac{2 \Gamma\left(1-\frac{k}{2 \kappa}\right)}{\Gamma\left(1-\frac{k}{2}\right)} \cos \left(\gamma k \frac{\pi}{2 \kappa}\right)=1
$$

Where $\gamma=K(\kappa)$.

After that we will obtain results given in Tables 1-4:

Table 1. Estimators of Index $k_{1}$ for a Case Where $\alpha_{0}=0,1$

\begin{tabular}{|c|c|c|c|c|c|c|}
\hline$\kappa$ & 0.4 & 0.5 & 0.6 & 0.7 & 0.8 & 0.9 \\
\hline$k_{1}$ & 0.717 & 0.9022 & 1.089 & 1.278 & 1.2799 & 1.284 \\
\hline
\end{tabular}

Table 2. Estimators of Index $k_{1}$ for a Case Where $\alpha_{0}=0,2$

\begin{tabular}{|c|c|c|c|c|c|c|}
\hline$\kappa$ & 0.4 & 0.5 & 0.6 & 0.7 & 0.8 & 0.9 \\
\hline$k_{1}$ & 0.6152 & 0.795 & 0.958 & 1.125 & 1.3 & 1.3016 \\
\hline
\end{tabular}

Table 3. Estimators of Index $k_{1}$ for a Case Where $\alpha_{0}=0,3$

\begin{tabular}{|c|c|c|c|c|c|c|}
\hline$\kappa$ & 0.4 & 0.5 & 0.6 & 0.7 & 0.8 & 0.9 \\
\hline$k_{1}$ & 0.697 & 0.613 & 0.642 & 0.688 & 0.6467 & 0.6425 \\
\hline
\end{tabular}

Table 4. Estimators of Index $k_{1}$ for a Case Where $\alpha_{0}=0,4$

\begin{tabular}{|c|c|c|c|c|c|c|}
\hline$\kappa$ & 0.4 & 0.5 & 0.6 & 0.7 & 0.8 & 0.9 \\
\hline$k_{1}$ & 0.6402 & 0.5816 & 0.635 & 0.7595 & 0.903 & 0.9282 \\
\hline
\end{tabular}




\section{Conclusion}

The purpose of this paper is to analyze and discuss the possibilities and the limitations of tail index estimation. We have shown in this paper that, even without knowing in advance the distribution of the theoretical tail index, it is possible to achieve a reasonable tail index estimate for a wide set of distribution functions. This paper describes Kesten's theorem to prove regular variation of the solution to the stochastic recurrent equation, its application to prove the property of GARCH model regular variation. The property of regular variation of finite joint distribution of the GARCH model has been proven, heavy tail index of $\mathrm{ARCH}(1)$ model distribution with stable residuals has been estimated. We obtain that the index of the regular variation of $\mathrm{ARCH}(1)$ model with residuals having a $\kappa$-stable distribution, $\varepsilon_{1}^{2} \sim S_{\kappa}(1,0,0)$.

\section{Acknowledgements}

This work was financially supported by Natural Science Foundation of Heilongiiang Province of China (Grant No.A201301).

\section{References}

[1] E. Brodin and C. Kluppelberg, "Etreme value theory in finace", Encyclopedia of quantitive risk assessment, (2007).

[2] A. L. Barabasi, "The orgin of bursts and heavy tails in human dynamics", Nature, vol. 5, (2005), pp. 207-211.

[3] C. H. Hailong, L. Chunli and Y. Chengji, "Research on Parmeters estimation and Application of AlphaStable Distributions", Journal of Harbin University of Science and Technology, vol. 8, (2013), pp. 63-68.

[4] B. A. Hill, "A simple approach to inference about the tail of a distribution", The Annals of Statistics, vol. 5, (1975), pp. 1163-1174.

[5] H. Kesten, "Random difference equations and renewal theory for products of random matrices", H. Kesten , Acta Math, vol. 11, (1973), pp. 207-248.

[6] C. M. Goldie, Implicit renewal theory and tails of solutions of random equations / C. M. Goldie // Ann. of appl. probab., vol. 2, (1991), pp. 126-166.

[7] B. Basrak, "Regular variation of GARCH processes / B. Basrak, R. A. Davis, T. Mikosch", Stochastic Process Appl., vol. 5, (2002), pp. 95-115.

[8] L. Breiman, "On some limit theorems similar to the arc-sin law, L. Breiman", Theory Probab. Appl. vol. 10, (1965), pp. 323-331.

[9] E. Iglesias, "Estimation of Tail Thickness Parameters from GARCH Models", E. Iglesias, Workshop Schedule Fall, Area Econometrics, Michigan State Univer sity. - [Electronic resource]. - 2007.http://www.indiana.edu/ econdept/workshops/Fall_2007_Papers / Iglesias_iltail59.pdf. - Date of access: 03.07, (2008).

[10] C. Stărică, "The Tales the Tails of GARCH, $(1,1)$ Processes Tell”, C. Stărică. -Mimeo: Chalmers University, vol. 531, (1999).

[11] N. Wagner, "Measuring tail thickness under GARCH and an application to extreme exchange rate changes / N. Wagner, T. A. Marsh", // Journal of empirical finance, vol. 12, (2005), pp.165-185. 
International Journal of Signal Processing, Image Processing and Pattern Recognition Vol. 10, No. 1 (2017) 